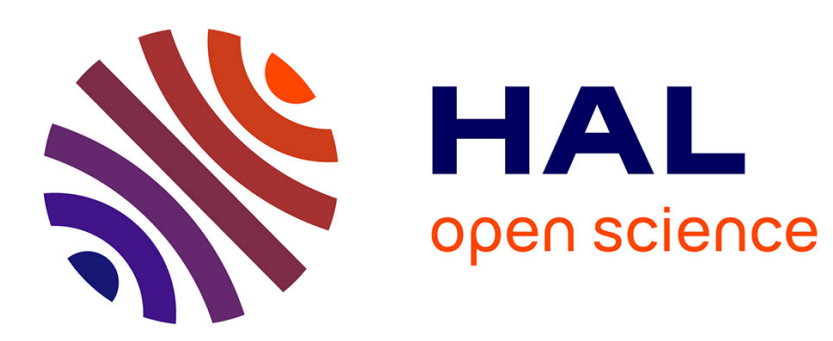

\title{
A Green Approach to Femtocells Capacity Improvement by Recycling Wasted Resources
}

Leonardo S. Cardoso, Marco Maso, Mérouane Debbah

\section{To cite this version:}

Leonardo S. Cardoso, Marco Maso, Mérouane Debbah. A Green Approach to Femtocells Capacity Improvement by Recycling Wasted Resources. WCNC 2013, Apr 2013, Shanghai, China. pp.3817 3822, 10.1109/WCNC.2013.6555183 . hal-00923455

\section{HAL Id: hal-00923455 \\ https://hal-centralesupelec.archives-ouvertes.fr/hal-00923455}

Submitted on 8 Jan 2014

HAL is a multi-disciplinary open access archive for the deposit and dissemination of scientific research documents, whether they are published or not. The documents may come from teaching and research institutions in France or abroad, or from public or private research centers.
L'archive ouverte pluridisciplinaire $\mathbf{H A L}$, est destinée au dépôt et à la diffusion de documents scientifiques de niveau recherche, publiés ou non, émanant des établissements d'enseignement et de recherche français ou étrangers, des laboratoires publics ou privés. 


\title{
A Green Approach to Femtocells Capacity Improvement by Recycling Wasted Resources
}

\author{
Leonardo S. Cardoso ${ }^{\diamond}$, Marco Maso ${ }^{\star, \dagger}$ and Mérouane Debbah ${ }^{\star}$ \\ ${ }^{\diamond}$ INRIA, Université de Lyon, INSA-Lyon, CITI-INRIA, France \\ ${ }^{\star}$ Alcatel-Lucent Chair in Flexible Radio, Supélec, France \\ $\dagger$ Department of Information Engineering, University of Padova, Italy \\ leonardo.sampaio-cardoso@inria.fr, \{marco.maso,merouane.debbah\}@ supelec.fr
}

\begin{abstract}
In this contribution we propose a method to increase the energy efficiency of orthogonal frequency division multiplexing (OFDM)-based femtocells. This is accomplished with no impact to the current power consumption, radio frequency (RF) circuitry, link adaptation strategies, bandwidth and transmit power. The proposed technique recycles redundant resources of OFDM transmissions (e.g., guard bands and cyclic prefixes), introduced to combat frequency selectivity. We borrow the underlying idea from a technique called cognitive interference alignment (CIA). Interestingly, our novel approach does not suffer from the same issues inherent to CIA, such as synchronization at the primary receiver and channel knowledge related complications. Nevertheless, it introduces a new issue related to the interference from the OFDM signal, which prompted the adoption of an adequate linear receiver at the femtocell user equipment. Numerical findings demonstrate that spectral efficiency gains are achieved, improving the energy efficiency of the femtocell by up to $20 \%$ for the simulated scenario.
\end{abstract}

Index Terms-Femtocells, green networks, energy efficiency, spectrum sharing, interference management, linear precoding

\section{INTRODUCTION}

The design of new network paradigms to overcome the limitations of $3 \mathrm{G}$ networks has been one of the most challenging tasks telecommunications researchers have faced during the last decade. Solutions, such as long term evolution advanced (LTE-A) [1], have been proposed to enhance the performance of current networks and meet the ever-growing user data demand. Yet, mobile data traffic is expected to increase 18-fold between 2011 and 2016 [2]. Without a change in network design, the risk of capacity shortfall or insufficient coverage could arise again. New technologies will likely be necessary to avoid a network breakdown.

One of the most promising strategies to increase the flexibility and robustness of next generation networks is believed to be a hierarchical base station deployment [3]. In fact, recent proposals point to complementing the legacy tier of macro-cells with a tier of low-power femtocell base stations. This way, a better average link quality, more efficient usage of spectrum resources and higher spatial reuse (co-channel deployment) could be brought to the network [3]. The current femtocell frenzy is motivated by the fact that they are good candidates for groundbreaking physical layer techniques, such as network multiple-input multiple-output (MIMO) [4] and dynamic spectrum access (DSA) [5].
Forecasted by the SMART 2020 report [6], femtocells should not only lead to performance enhancement, but also operate in a more energy efficient and green way [7]. In the same spirit, the LTE standard core has included several energy efficient techniques, such as bandwidth reduction, carrier aggregation strategies and cell switch-off approaches, just to name a few [8]. At the network level, self-organizing techniques enable traffic demand tracking, aiming at a reduction of the energy expenses [9]. Otherwise, at the device level, link adaptation strategies for OFDM transmissions have been studied and proposed. Such approaches involve a design shift in both RF circuitry and resource allocation strategies to improve energy efficiency [10].

In this contribution, we propose a new device level technique to increase the energy efficiency for legacy OFDM-based femtocells, by enhancing spectral efficiency while preserving power consumption. Unlike currently proposed approaches [8], [10], we show that spectral efficiency can be improved without changing the hardware design, link adaptation, bandwidth or transmit power. Our power efficient approach recycles the redundant resources of OFDM transmissions (i.e., guard bands or cyclic prefixes) introduced to combat the frequency selectivity. Furthermore, our approach can be implemented alongside current proposals, adding up the total energy efficiency.

We borrow concepts from overlay DSA and cognitive radio (CR) [5]. Our technique is based on cognitive interference alignment (CIA), recently introduced in [11] to address the $\mathrm{CR}$ interference channel problem. In its classical form, CIA allows a cognitive transmitter to serve a secondary user by sharing the spectrum with a licensee OFDM primary macro-cell, protecting the primary OFDM receiver from undesired interference. Herein, we exploit the flexibility of CIA to design a clever hybrid transceiver that simultaneously sends both primary and secondary signals. A femtocell scenario is considered in our analysis. Note that the validity of the proposed approach is not restricted to femtocells, and can be seamlessly extended to any interference mitigation scenario. Unlike [11], this hybrid approach does not suffer from some issues inherent to CIA, such as synchronization at the primary receiver and interference channel knowledge acquisition. On the other hand, the simultaneous transmission of primary and secondary messages introduces a new challenge since the femtocell receiver is now subject to strong primary interference. 
This issue prompted the adoption of an appropriate linear receiver at the femtocell user equipment. Numerical findings demonstrate that energy efficiency enhancements are achieved due to the spectral efficiency gains, maintaining the same power at the femtocell.

This work is organised as follows. In Sec. II we briefly review CIA and discuss about its limitations. Then, in Sec. III, we introduce the adopted channel model. In Sec. IV, we show the receiver structure and discuss the performance of the overall scheme. In Sec. V we present the numerical results. Finally, the conclusions and further research directions are given in Sec. VI.

\section{Cognitive Interference Alignment}

Let us start, by reviewing the conventional CIA [11]. In it, a primary (OFDM-based) and a secondary system share the spectrum at the same time avoiding interference to the primary receiver. This is achieved by means of intelligent precoding at the secondary transmitter, that encodes the signal on the nullspace of the channel to the primary receiver. To understand CIA's structure, let us start by taking a two-user model. To clarify the notation, wherever present, the subscript " $p$ " refers to the primary system and the subscript "s" refers to the secondary system. Furthermore, given a vector $\mathbf{a}=\left(a_{1}, \ldots, a_{N}\right)$, we denote as $\mathrm{d}(\mathbf{a})$ a diagonal matrix such that $[\mathrm{d}(\mathbf{a})]_{i, i}=a_{i}$, for the sake of compactness of the notation. Additionally, we define $\mathbf{I}_{N}$ and $\mathbf{0}_{N \times M}$ as the identity matrix of size $N \times N$, and the all-zeros matrix of size $N \times M$, respectively.

The primary system adopts OFDM time division duplex (TDD), with $N$ carriers and an $L$-sized cyclic prefix (CP), for a block size of $N+L$. The secondary adopts CIA, with block size of $N+L$. The participating channels $\mathbf{h}^{(\mathrm{p}, \mathrm{p})}, \mathbf{h}^{(\mathrm{p}, \mathrm{s})}, \mathbf{h}^{(\mathrm{s}, \mathrm{p})}$, $\mathbf{h}^{(\mathrm{s}, \mathrm{s})} \in \mathcal{C} \mathcal{N}\left(0, \mathbf{I}_{l+1} /(l+1)\right)$ are i.i.d. Rayleigh fading channel vectors of size $l+1$ taps. We define $\mathbf{s}_{\mathrm{p}} \in \mathcal{C} \mathcal{N}\left(0, \mathrm{~d}\left(\mathbf{p}_{\mathrm{p}}\right)\right)$ and $\mathbf{s}_{\mathrm{s}} \in \mathcal{C} \mathcal{N}\left(0, \mathrm{~d}\left(\mathbf{p}_{\mathrm{s}}\right)\right)$ as the input symbol vector for the primary and secondary system, of size $N$ and $L$ respectively. Note that, $\mathbf{p}_{\mathrm{p}}=\left(p_{\mathrm{p}, 1}, \ldots, p_{\mathrm{p}, N}\right) \in \mathbb{R}^{N}$ and $\mathbf{p}_{\mathrm{s}}=\left(p_{\mathrm{s}, 1}, \ldots, p_{\mathrm{s}, L}\right) \in \mathbb{R}^{L}$ are power allocation vectors with $p_{\mathrm{a}, i}$ power of the $i^{\text {th }}$ input symbol at the transmitter "a". Let the received signal at the primary and secondary user's antennas be

$$
\begin{aligned}
& \mathbf{y}_{\mathrm{p}}=\mathbf{H}_{\mathrm{pp}} \mathbf{A} \mathbf{F}^{-1} \mathbf{s}_{\mathrm{p}}+\mathbf{H}_{\mathrm{sp}} \mathbf{E s}_{\mathrm{s}}+\mathbf{n}_{\mathrm{p}} \\
& \mathbf{y}_{\mathrm{s}}=\mathbf{H}_{\mathrm{ss}} \mathbf{E s}_{\mathrm{s}}+\mathbf{H}_{\mathrm{ps}} \mathbf{A} \mathbf{F}^{-1} \mathbf{s}_{\mathrm{p}}+\mathbf{n}_{\mathrm{s}}
\end{aligned}
$$

respectively, where $\mathbf{F} \in \mathbb{C}^{N \times N}$ is a unitary discrete Fourier transform (DFT) matrix with $[\mathbf{F}]_{(k+1, l+1)}=\frac{1}{\sqrt{N}} e^{-i 2 \pi \frac{k l}{N}}$ for $k, l=\{0, \ldots, N-1\}$,

$$
\mathbf{A}=\left[\begin{array}{rr}
\mathbf{0}_{L, N-L} & \mathbf{I}_{L} \\
\mathbf{I}_{N} &
\end{array}\right]
$$

is a $\mathrm{CP}$ insertion matrix of size $(N+L) \times N$, $\mathbf{E} \in \mathbb{C}^{(N+L) \times L}$ is the CIA precoder matrix, and $\mathbf{n}_{\mathrm{s}}, \quad \mathbf{n}_{\mathrm{p}} \sim \operatorname{eN}\left(0, \sigma^{2} \mathbf{I}_{N+L}\right)$ are additive white Gaussian noise (AWGN) vectors. Note that, in (1) and (2),
$\mathbf{H}_{\mathrm{ab}} \in \mathbb{C}^{(N+L) \times(N+L)}$ are matrices modeling the convolution of the channel from "a" to "b" with the signal, given by

$$
\mathbf{H}_{\mathrm{ab}}=\left[\begin{array}{ccccccc}
h_{0}^{(\mathrm{a}, \mathrm{b})} & 0 & \cdots & 0 & h_{l}^{(\mathrm{a}, \mathrm{b})} & \cdots & h_{1}^{(\mathrm{a}, \mathrm{b})} \\
\vdots & \ddots & \ddots & \ddots & \ddots & \ddots & \vdots \\
\vdots & \ddots & \ddots & \ddots & \ddots & \ddots & h_{l-1}^{(\mathrm{a}, \mathrm{b})} \\
h_{l}^{(\mathrm{a}, \mathrm{b})} & \cdots & \cdots & h_{0}^{(\mathrm{a}, \mathrm{b})} & 0 & \cdots & 0 \\
0 & \ddots & \ddots & \ddots & \ddots & \ddots & \vdots \\
\vdots & \ddots & \ddots & \ddots & \ddots & \ddots & 0 \\
0 & \ddots & 0 & h_{l}^{(\mathrm{a}, \mathrm{b})} & \cdots & \cdots & h_{0}^{(\mathrm{a}, \mathrm{b})}
\end{array}\right] .
$$

In the CIA scenario, we aim at canceling the secondary transmitter's message at the primary receiver. With CP removal and DFT of the signal at the primary receiver we get

$$
\mathbf{r}_{\mathrm{p}}=\mathbf{F B} \mathbf{y}_{\mathrm{p}}=\mathbf{F} \overline{\mathbf{H}}_{\mathrm{pp}} \mathbf{A} \mathbf{F}^{-1} \mathbf{s}_{\mathrm{p}}+\mathbf{F} \overline{\mathbf{H}}_{\mathrm{sp}} \mathbf{E} \mathbf{s}_{\mathrm{s}}+\mathbf{F} \overline{\mathbf{n}}_{\mathrm{p}}
$$

where $\mathbf{B}=\left[\mathbf{0}_{N \times L} \mathbf{I}_{N}\right]$ is a $\mathrm{CP}$ removal matrix of size $N \times(N+L)$, the $\overline{\mathbf{H}}_{\mathrm{ab}} \in \mathbb{C}^{N \times(N+L)}$ matrices are the $\mathbf{H}_{\mathrm{ab}}$ matrices stripped off the $L$ first lines and $\mathbf{F} \overline{\mathbf{n}}_{\mathrm{p}}$ has size $N$ and the same statistics of $\mathbf{n}_{\mathrm{p}}$.

The interference cancelation constraint at the CIA transmitter is

$$
\overline{\mathbf{H}}_{\mathrm{sp}} \mathbf{E}=\mathbf{0}_{N \times L},
$$

for all $\mathbf{s}_{\mathrm{s}}$, under the restriction that $\mathbf{E} \neq \mathbf{0}_{(N+L) \times L}$. We have shown in [11] that, for perfect receiver synchronization and perfect channel state information at the secondary transmitter (CSIT), the optimal CIA precoder is given by a semi-unitary matrix structure such that

$$
\operatorname{span}(\mathbf{E})=\operatorname{ker}\left(\overline{\mathbf{H}}_{\mathrm{sp}}\right),
$$

followed by an appropriate water-filling power allocation [12]. Reevaluating (1) in the light of (4), we obtain

$$
\mathbf{y}_{\mathrm{p}}=\mathbf{H}_{\mathrm{pp}} \mathbf{x}_{\mathrm{p}}+\left[\begin{array}{c}
\mathbf{K} \\
\mathbf{0}_{N \times L}
\end{array}\right] \mathbf{s}_{\mathrm{s}}+\mathbf{n}_{\mathrm{p}},
$$

where $\mathbf{K} \in \mathbb{C}^{L \times L}$ is a matrix whose constant size $L$ depends only on $\operatorname{dim} \operatorname{ker}\left(\overline{\mathbf{H}}_{\mathrm{sp}}\right)$. Recomputing $\mathbf{r}_{\mathrm{p}}$ from (3), we get

$$
\mathbf{r}_{\mathrm{p}}=\mathbf{F} \overline{\mathbf{H}}_{\mathrm{pp}} \mathbf{A} \mathbf{F}^{-1} \mathbf{s}_{\mathrm{p}}+\mathbf{F} \overline{\mathbf{n}}_{\mathrm{p}},
$$

from where we clearly see that the primary system sees no interference from the secondary system. This result is valid for perfect synchronization at the primary receiver and perfect CSIT w.r.t. $\mathbf{h}^{(\mathrm{s}, \mathrm{p})}$. If one or both assumptions are not verified, the effectiveness of CIA would be highly reduced as we discuss in the following.

\section{A. Synchronization}

As shown in (5), CIA's interference cancelation is completely dependent on OFDM's CP removal, where $\mathbf{K}$ is dropped, making the whole interference term $\mathbf{F} \overline{\mathbf{H}}_{\mathrm{sp}} \mathbf{E} \mathbf{s}_{\mathrm{s}}=\mathbf{0}_{N}$. Hence, if the signal is poorly synchronized at sample level at the receiver, the $\mathrm{CP}$ removal will discard the wrong part of the signal. Indeed, the importance of perfect synchronization at the receiver is not only crucial for interference cancelation in CIA, 
but to any null-space precoder that exploits the redundancy in OFDM, e.g., Vandermonde-subspace frequency division multiplexing (VFDM) for which a comprehensive discussion is given in [13]. In other words, significant interference may be experienced at the primary receiver in case of inaccurate synchronization, growing rapidly with the number of unsynchronized samples [13].

\section{B. Channel Estimation Issue}

Another crucial aspect of CIA is the CSIT acquisition at the secondary transmitter w.r.t. $\mathbf{h}^{(\mathrm{s}, \mathrm{p})}$. Two main issues can be identified. Firstly, the secondary transmitter must know the primary system's channel estimation procedure, to acquire the interference channel CSIT. Secondly, the quality of the CSIT highly depends on the signal to interference plus noise ratio (SINR) at the secondary transmitter (during the uplink) and on the channel's coherence time.

In TDD networks, the first issue may be addressed by exploiting the training/transmission procedure of the primary system, as described in [14]. For a block fading model with coherence time $\mathcal{T}$, channel estimations in the primary system are performed in a time $\tau \leq \mathcal{T}$. A CIA transmitter can thus acquire CSIT with periodicity $\mathcal{T}$, calculating $\mathbf{E}$ and engaging in transmission during a time $\mathcal{T}-\tau$.

The second issue depends on the characteristics of the operating scenario. For instance, in the case of low SINR at the transmitter, more training would be necessary to acquire a better quality channel estimation [14]. If the receivers in both systems are mobile user equipments, the coherence time of their channel would vary depending on the mobility pattern, speed, and changes in the surrounding environment, possibly reducing the time available for the channel estimations. Consequently, a secondary CIA transmitter operating in such scenarios would face very stringent time constraints. As a result, the whole feasibility of the precoder design could be compromised by wrong or outdated CSIT yielding imperfectly designed precoders and poor overall system performance.

A further discussion of CSIT acquisition at the secondary transmitter is also presented in [13], as well as a practical proposal for a channel estimation procedure.

\section{HYBRID OFDM - CIA TRANSMITTER}

Consider the layout presented in Fig. 1, where a single femtocell, adopted to extend coverage and capacity, serves both the primary and secondary users. As stated before, the femtocell device adopting OFDM introduces a redundancy to combat the frequency-selectivity of the channel. The CP is discarded at the primary receiver to avoid inter-block interference (IBI), and thus, all power invested in the CP is lost. This results in both spectral and energy inefficiencies. We aim at showing how these wasted resources can be compensated by the simultaneous transmission of a primary (OFDM) and a secondary (CIA) signal at the femtocell. As seen in Sec. II, CIA provides $L$ additional transmit dimensions (equivalent to the CP size in OFDM), while preserving OFDM's $N$ interferencefree information symbols. Thus, a total of $N+L$ information

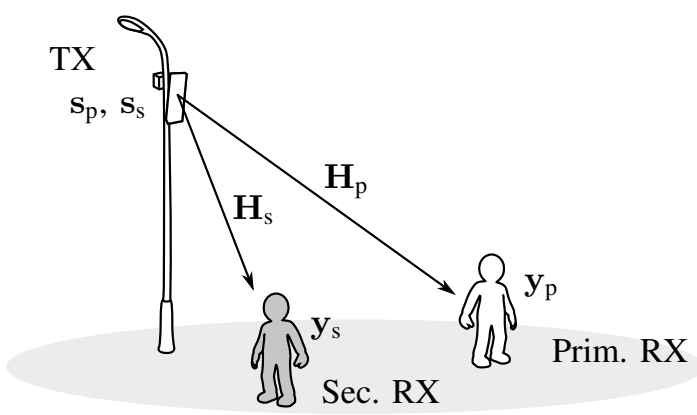

Fig. 1. Layout for simultaneous primary and secondary transmissions.

symbols can be sent in a simultaneous transmission. We note that, this does not require the installation of an additional transmitter or antenna, as for the conventional CIA.

Making a parallel with conventional CIA, herein we have that $\mathbf{H}_{\mathrm{ss}}=\mathbf{H}_{\mathrm{ps}}=\mathbf{H}_{\mathrm{s}}$, and $\mathbf{H}_{\mathrm{pp}}=\mathbf{H}_{\mathrm{sp}}=\mathbf{H}_{\mathrm{p}}$. Moreover, we define $\overline{\mathbf{H}}_{\mathrm{p}}=\overline{\mathbf{H}}_{\mathrm{pp}}$ to further simplify the notation. Let $\Delta^{(l)}(\cdot)$ and $\Delta^{(u)}(\cdot)$ be two operators that extract the lower and the upper triangular part of a matrix argument, respectively. We can rewrite the received signals at both the primary and secondary receivers as follows

$$
\begin{aligned}
\mathbf{r}_{\mathrm{p}} & =\mathbf{F} \overline{\mathbf{H}}_{\mathrm{p}} \mathbf{A} \mathbf{F}^{-1} \mathbf{s}_{\mathrm{p}}+\mathbf{F} \overline{\mathbf{H}}_{\mathrm{p}} \mathbf{E} \mathbf{s}_{\mathrm{s}}+\mathbf{F} \overline{\mathbf{n}}_{\mathrm{p}} \\
\mathbf{r}_{\mathrm{s}} & =\mathbf{y}_{\mathrm{s}} \\
& =\left(\Delta^{(l)}\left(\mathbf{H}_{\mathrm{s}}\right)+\Delta^{(u)}\left(\mathbf{H}_{\mathrm{s}}\right)\right) \mathbf{E} \mathbf{s}_{\mathrm{s}}+\mathbf{H}_{\mathrm{s}} \mathbf{A} \mathbf{F}^{-1} \mathbf{s}_{\mathrm{p}}+\mathbf{n}_{\mathrm{s}},
\end{aligned}
$$

where, differently from [11], the secondary receiver does not discard the first $L$ received symbols leading us to let $\mathbf{r}_{\mathrm{s}}=\mathbf{y}_{\mathrm{s}}$. Additionally, in (7), a decomposition of the channel for the CIA transmission into two components $\Delta^{(l)}\left(\mathbf{H}_{\mathrm{s}}\right)$ and $\Delta^{(u)}\left(\mathbf{H}_{\mathrm{s}}\right)$ represents the contribution of the channel that generates inter-symbol interference (ISI) and IBI, respectively [15]. Since the secondary receiver does not discard the CP, the IBI is not eliminated and has to be taken into account into the model. We recall that, $\mathbf{E}$ can be created to satisfy (3), like in the conventional CIA case [11], thanks to the knowledge of $\overline{\mathbf{H}}_{\mathrm{p}}$ available at the hybrid transmitter. In [16], a similar scenario is adopted for physical layer security, where a Vandermonde precoder is used to transmit a common and a private message to two different receivers. In spite the fact that our hybrid transmitter structure is similar, we do not need to enforce secure communications since our focus is on energy efficiency. Therefore, we do not encode all messages with the nullspace precoder nor discard the $\mathrm{CP}$ in our reception strategy. These characteristics are at the heart of CIA, a more robust technique w.r.t. diverse power delay profile (PDP) configurations, than [16].

We note that, from the primary receiver's point of view, the interfering and useful channels coincide. Nevertheless, interference and useful signals are subject to different precoding strategies, yielding two independent equivalent channel representations. Thus, only the secondary message is canceled, with no effect whatsoever to the primary reception. Likewise, the secondary channel is the same for the intended message and interference, a fact that impacts the performance of the secondary system. We will see in the following how the 
secondary receiver can cope with the issue, by means of an appropriate linear equalizer.

Now, let $\mathbf{x}_{\mathrm{p}}=\mathbf{A F ^ { - 1 }} \mathbf{s}_{\mathrm{p}}$ and $\mathbf{x}_{\mathrm{s}}=\mathbf{E} \mathbf{s}_{\mathrm{s}}$, both of size $N+L$, be the primary and secondary signal components at the femtocell's antenna. Let us assume that the power budget for the legacy OFDM-based transmission at the femtocell is $P$. Then, if we let $\mathbf{x}=\mathbf{x}_{\mathrm{p}}+\mathbf{x}_{\mathrm{s}}$ be the hybrid femtocell overall transmit vector, we have that in order to maintain the same power consumption as the OFDM case, it must hold that

$$
\operatorname{tr}\left(\mathbb{E}\left[\mathbf{x x}^{\mathrm{H}}\right]\right) \leq P .
$$

In the proposed scheme, both primary and secondary messages are transmitted at the same time, hence they share the transmitter's power budget. We note that, while the CIA precoder is designed to protect the primary receiver from undesired interference, the converse is not true for the OFDM transmission w.r.t. the secondary receiver. Neither deterministic nor stochastic information about this interference is available at the hybrid transmitter. In fact, $\mathbf{H}_{\mathrm{s}}$ is a finite dimension Toeplitz matrix, whose eigenvalue and eigenvector distribution is not currently known. As a consequence, analytic optimization of the power splitting strategy between the two transmissions is not feasible, and only numerical iterative approaches could be adopted to solve it. Then, since the femtocell won't be able to find the optimal power splitting strategy due to computational and time constraints, we assume that it will statically split the maximum power between the two transmissions. Accordingly, $\mathbf{x}_{\mathrm{p}}$ and $\mathbf{x}_{\mathrm{s}}$ are derived disjointly, such that

$$
\begin{aligned}
\operatorname{tr}\left(\mathbb{E}\left[\mathbf{x}_{\mathrm{p}} \mathbf{x}_{\mathrm{p}}^{\mathrm{H}}\right]\right) & \leq P_{\mathrm{p}} \\
\operatorname{tr}\left(\mathbb{E}\left[\mathbf{x}_{\mathrm{s}} \mathbf{x}_{\mathrm{s}}^{\mathrm{H}}\right]\right) & \leq P_{\mathrm{s}} \\
P_{\mathrm{p}}+P_{\mathrm{s}} & =P
\end{aligned}
$$

where the optimal power loading strategy for both cases [11], [12] is adopted separately. We remark that, in general, such an approach will induce a signal to noise ratio (SNR) loss w.r.t. the legacy standalone OFDM femtocell's transmission. The impact of this loss on the performance of the system will be analyzed in Sec. V, where a study on the spectral efficiency maximizing power splitting strategy is provided.

Finally, we note that this hybrid transmitter design addresses the issues affecting CIA discussed in Sec. II-A and II-B. In fact, since both the OFDM and CIA messages are transmitted simultaneously and experience the same channel, synchronization discrepancies at the receiver are always avoided. In other words, the OFDM and CIA messages will always be synchronized at the primary receiver at sample level, regardless of the adopted time synchronization algorithm. Similarly, concerning the CSIT, no adaptive procedure is required at the hybrid transmitter to design $\mathbf{E}$. This is due to the aforementioned equivalence $\mathbf{H}_{\mathrm{sp}}=\mathbf{H}_{\mathrm{ps}}$, considerably increasing the feasibility of the precoder design even in case of high mobility scenarios.

\section{LINEAR EQUALIZER}

As described before, one of the most striking differences between the hybrid scheme proposed herein and the conventional CIA, is the fact that the secondary receiver's physical interference and main channel coincide, i.e., $\mathbf{H}_{\mathrm{s}}$. This issue imposes a tough interference cost, since full interference will always be seen at the secondary receiver, irrespective of its fading state. As such, the secondary receiver becomes the weak link of the technique, and needs to be addressed carefully. Before starting, let us consider (7) and rewrite the received signal at the secondary receiver as

$$
\mathbf{r}_{\mathrm{s}}=\widetilde{\mathbf{H}}_{\mathrm{s}}^{(l)} \mathbf{s}_{\mathrm{s}}+\widetilde{\mathbf{H}}_{\mathrm{s}}^{(u)} \mathbf{s}_{\mathrm{s}}+\widetilde{\mathbf{H}}_{\mathrm{s}}^{(p)} \mathbf{s}_{\mathrm{p}}+\mathbf{n}_{\mathrm{s}},
$$

where $\widetilde{\mathbf{H}}_{\mathrm{s}}^{(l)}=\Delta^{(l)}\left(\mathbf{H}_{\mathrm{s}}\right) \mathbf{E}, \widetilde{\mathbf{H}}_{\mathrm{s}}^{(u)}=\Delta^{(u)}\left(\mathbf{H}_{\mathrm{s}}\right) \mathbf{E} \in \mathbb{C}^{(N+L) \times L}$ are equivalent representations of the ISI and IBI channel contribution, respectively. $\widetilde{\mathbf{H}}_{\mathrm{s}}^{(p)}=\mathbf{H}_{\mathrm{s}} \mathbf{A} \mathbf{F}^{-1} \in \mathbb{C}^{(N+L) \times N}$ is an the equivalent channel matrix related to the primary transmission contribution at the CIA receiver.

We know from [12], that the linear equalizer that maximizes the output SINR for any variance of the Gaussian noise is the so called minimum mean square error (MMSE) receiver. Therefore, let us assume that the secondary receiver possesses perfect CSI w.r.t. to the equivalent channel matrices, by means of a channel estimation made possible by the TDD structure [14]. Accordingly, the experienced interference plus noise component is obtained as $\boldsymbol{\eta}_{\mathrm{s}}=\widetilde{\mathbf{H}}_{\mathrm{s}}^{(p)} \mathbf{s}_{\mathrm{p}}+\widetilde{\mathbf{H}}_{\mathrm{s}}^{(u)} \mathbf{s}_{\mathrm{s}}+\mathbf{n}_{\mathrm{s}}$, with covariance matrix $\mathbf{S}_{\eta}=\mathbb{E}\left[\boldsymbol{\eta}_{\mathrm{s}} \boldsymbol{\eta}_{\mathrm{s}}^{\mathrm{H}}\right]$. Then, the MMSE equalizer $\mathbf{C}_{\mathrm{s}}$ can be derived as [12]

$$
\mathbf{C}_{\mathrm{s}}=\widetilde{\mathbf{H}}_{\mathrm{s}}^{(l) \mathrm{H}}\left(\mathbf{S}_{\eta}+\widetilde{\mathbf{H}}_{\mathrm{s}}^{(l)} \widetilde{\mathbf{H}}_{\mathrm{s}}^{(l) \mathrm{H}}\right)^{-1}
$$

The estimated symbols at the secondary receiver can be obtained as

$$
\hat{\mathbf{s}}_{\mathrm{s}}=\mathbf{C}_{\mathrm{s}} \mathbf{r}_{\mathrm{s}}
$$

resulting in an effective SINR of the $k^{t h}$ decoded symbol of

$$
\begin{gathered}
\gamma_{\mathrm{s}, k}=p_{\mathrm{s}, k} \widetilde{\mathbf{h}}_{k}^{(l) \mathrm{H}}\left(\widetilde{\mathbf{H}}_{\mathrm{s}}^{(u)} d\left(\mathbf{p}_{\mathrm{s}}\right) \widetilde{\mathbf{H}}_{\mathrm{s}}^{(u) \mathrm{H}}+\widetilde{\mathbf{H}}_{\mathrm{s}}^{(p)} d\left(\mathbf{p}_{\mathrm{p}}\right) \widetilde{\mathbf{H}}_{\mathrm{s}}^{(p) \mathrm{H}}+\right. \\
\left.\widetilde{\mathbf{H}}_{\mathrm{s},[k]}^{(u)} d\left(\mathbf{p}_{\mathrm{s},[k]}\right) \widetilde{\mathbf{H}}_{\mathrm{s},[k]}^{(u) \mathrm{H}}+\sigma^{2} \mathbf{I}_{N+L}\right)^{\dagger} \widetilde{\mathbf{h}}_{k}^{(l)},
\end{gathered}
$$

where, for the sake of compactness, $\widetilde{\mathbf{H}}_{\mathrm{s}}^{(l)}=\left[\widetilde{\mathbf{h}}_{1}^{(l)}|\ldots| \widetilde{\mathbf{h}}_{L}^{(l)}\right]$, $\widetilde{\mathbf{H}}_{\mathrm{s},[k]}^{(u)}=\left[\begin{array}{ll|l|l|l|l|l|}\widetilde{\mathbf{h}}_{1}^{(u)} & \ldots & \widetilde{\mathbf{h}}_{k-1}^{(u)} \mid \widetilde{\mathbf{h}}_{k+1}^{(u)} & \ldots & \widetilde{\mathbf{h}}_{L}^{(u)}\end{array}\right]$ and $\mathbf{p}_{\mathrm{s},[i]}=\left[p_{\mathrm{s}, 1}, \ldots, p_{\mathrm{s}, k-1}, p_{\mathrm{s}, k+1}, \ldots, p_{\mathrm{s}, L}\right]$. Accordingly, the spectral efficiency of the CIA transmission can be computed as

$$
R_{\mathrm{s}}=\frac{1}{N+L} \sum_{k=1}^{L} \log _{2}\left(1+\gamma_{\mathrm{s}, k}\right) .
$$

Looking back at the primary receiver, we remark that thanks to adoption of CIA's precoder $\mathbf{E}$, no interference is generated by the secondary transmission and the OFDM receiver can apply the decoding procedure adopted in classical OFDM systems. As a consequence, a zero-forcing $(\mathrm{ZF})$ equalizer is adopted to obtain an estimate of the received symbol vector $\hat{\mathbf{s}}_{\mathrm{p}}$, and the corresponding spectral efficiency $R_{\mathrm{p}}$ can be computed as detailed in [12]. Then, the overall spectral efficiency of the hybrid femtocell's transmission is computed as $R_{\mathrm{h}}=R_{\mathrm{p}}+R_{\mathrm{s}}$. 


\section{Numerical Results}

In this section we evaluate the performance of the proposed hybrid transceiver by means of extensive Monte Carlo simulations. The spectral efficiency of a legacy OFDM-based femtocell is taken as a reference to assess the performance of the proposed approach. For the simulations, we assume $N=128$ subcarriers and a CP of $L=32$, as one of LTE-A's configurations [1]. In the simulations, noise is generated w.r.t. the average SNR per symbol of our reference legacy OFDM system, equivalent to set $P_{p}=P$ and $P_{s}=0$. The average SNR (in $\mathrm{dB}$ ) of the $\mathrm{i}^{\text {th }}$ received symbol is given by

$$
\mathrm{SNR}_{i}=\log _{10} \mathbb{E}\left[\frac{p_{\mathrm{p}, i} \overline{\mathbf{h}}_{p, i}^{\mathrm{H}} \overline{\mathbf{h}}_{p, i}}{\sigma^{2}}\right]
$$

$\forall i \in[1, N], \sum_{i}^{N} p_{\mathrm{p}, i}=P$ and with $\overline{\mathbf{h}}_{p, i} i^{\text {th }}$ column of $\overline{\mathbf{H}}_{\mathrm{p}}$. For the hybrid system, we keep the same noise power $\left(\sigma^{2}\right)$, and split the total power $P$ for the OFDM and CIA transmission as in (11), such that we provide a fair comparison with the proposed legacy scheme. In other words, by ensuring the same noise level for both reference and hybrid cases, we take into account the SNR reduction experienced by the primary and secondary receivers w.r.t. the reference legacy OFDM femtocell receiver, due to the power splitting strategy adopted in the hybrid scheme.

Let $R_{\text {OFDM }}$ be the spectral efficiency of the OFDM transmission, and $\mathcal{E}_{\mathrm{OFDM}} \triangleq \frac{R_{\mathrm{OFDM}}}{P}$ its energy efficiency, measured in $\mathrm{bit} / \mathrm{s} / \mathrm{Hz} / \mathrm{W}$. If we define the energy efficiency of the hybrid transmission as $\mathcal{E}_{\mathrm{h}} \triangleq \frac{R_{\mathrm{h}}}{P}$, then the percent change in the energy efficiency experienced by the femtocell when switching from legacy OFDM to the proposed hybrid scheme $\xi \in \mathbb{R}$, can be defined as

$$
\xi=100\left(\frac{\varepsilon_{\mathrm{h}}}{\varepsilon_{\mathrm{OFDM}}}-1\right)=100\left(\frac{R_{\mathrm{h}}}{R_{\mathrm{OFDM}}}-1\right) .
$$

Thus, any change in spectral efficiency is translated into an equivalent change in energy efficiency, since the total transmit power remains the same. To numerically obtain the semiunitary $\mathbf{E}$, we let $\mathbf{V}=\left[\mathbf{v}_{1}\left|\mathbf{v}_{2}\right| \cdots \mid \mathbf{v}_{(N+L)}\right]$ be obtained from the singular value decomposition of $\mathbf{H}_{\mathrm{p}}=\mathbf{U} \boldsymbol{\Lambda} \mathbf{V}^{\mathrm{H}}$. Then we make $\mathbf{E}=\left[\mathbf{v}_{N+1}|\cdots| \mathbf{v}_{(N+L)-1} \mid \mathbf{v}_{N+L}\right]$. Other methods to obtain an equally optimal semi-unitary $\mathbf{E}$ can be found in [13].

Firstly, we analyze the effect of the hybrid's transmitter power splitting strategy on the energy efficiency of the femtocell. For this analysis, all channel vectors are defined as in Sec. II, with $l=L$ number of channel taps and uniform PDP. To determine the best $\xi$ we let $P_{\mathrm{p}} / P$ vary from 0.5 to 1 and adjust $P_{\mathrm{s}}$ accordingly. In Fig. 2 the best value for $\xi$, obtained for $P_{\mathrm{p}} / P=0.87$, is of about $15 \%, 11 \%$ and $4 \%$ for the SNRs of 35,20 and $5 \mathrm{~dB}$, respectively. This shows that the higher efficiency of an OFDM transmission w.r.t. CIA calls for an unbalanced power splitting in favor of the former, which can carry more information per block. On the other hand, evident energy efficiency enhancements are experienced at the optimal value $P_{\mathrm{p}} / P=0.87$ for the three considered SNR regimes. Remarkably, for a medium-to-high SNR regime, the hybrid approach yields a gain of $15 \%$ to the legacy standalone OFDM transmission. We note that, the for non-optimal values of $P_{p} / P, \xi$ experiences different trends. This is due to the unbalanced spectral efficiency contribution from the OFDM and CIA parts to the overall hybrid transmitter performance.

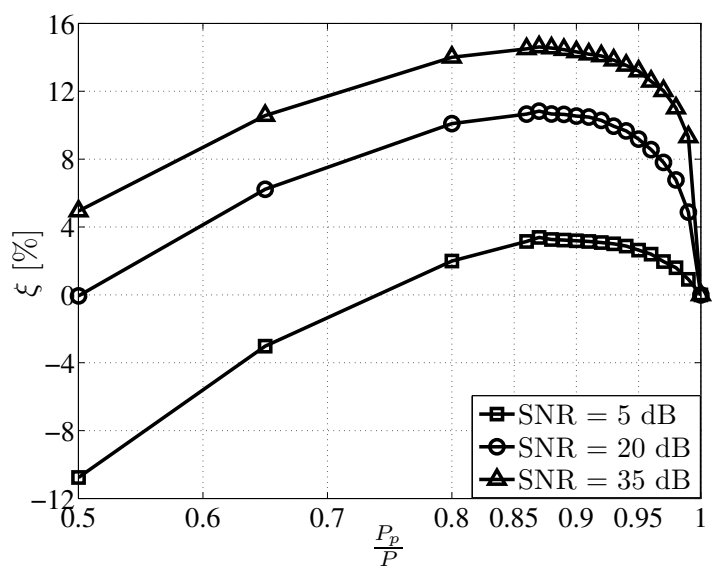

Fig. 2. Percent energy efficiency change w.r.t. the legacy OFDM femtocell for a uniform Rayleigh fading channel.

We know that uniform PDP channels are not realistic in nature. To grasp the effect of realistic channels on the performance of our hybrid transmitter, we analyzed the effect of an exponentially decreasing PDP for the considered Rayleigh fading channel. In the results that follow, we adopt a rather fast channel decay of $T_{s} / \tau_{d}=1.25$, where $T_{s}$ is the sample time and $\tau_{d}$ the root mean square delay spread of the channel. As before, we let the values of $P_{\mathrm{p}} / P$ vary from 0.5 to 1 and adjust $P_{\mathrm{s}}$ accordingly. In Fig. 3, we see that the best power splitting strategy is identical to the uniform PDP case, showing that the PDP has no evident influence on this criterion. Nevertheless the gains at medium-to-high SNR regime are accentuated (up to $20 \%$ for $35 \mathrm{~dB}$ ), while in the low SNR regime they disappear. This is due to CIA's behavior for exponential PDPs. In fact, as discussed in [11] for exponential PDPs, CIA is less efficient at low SNR due to a worse conditioning of $\mathbf{E}$, but more efficient at high SNR due to the lower IBI, experienced thanks to a smaller delay spread of the channel.

We have seen that the hybrid transmitter provides the best performance when the contribution from CIA adds up on the contribution of the OFDM transmission, occurring at a $P_{\mathrm{p}} / P$ of about 0.87 . In this final part, we focus on the best $P_{\mathrm{p}} / P$ and extend the SNR range, to understand how the gains of the hybrid scheme behave w.r.t. the standalone OFDM transmission for both PDP cases.

In Fig. 4 we see the performance of the hybrid scheme for both uniform and exponential PDP channels. Corroborating our previous findings, for the uniform PDP case the proposed scheme always provides gains, i.e., $\xi>0$, even when the SNR is as low as $0 \mathrm{~dB}$. Conversely, for the exponential PDP case, the hybrid approach experiences energy efficiency gains w.r.t. 


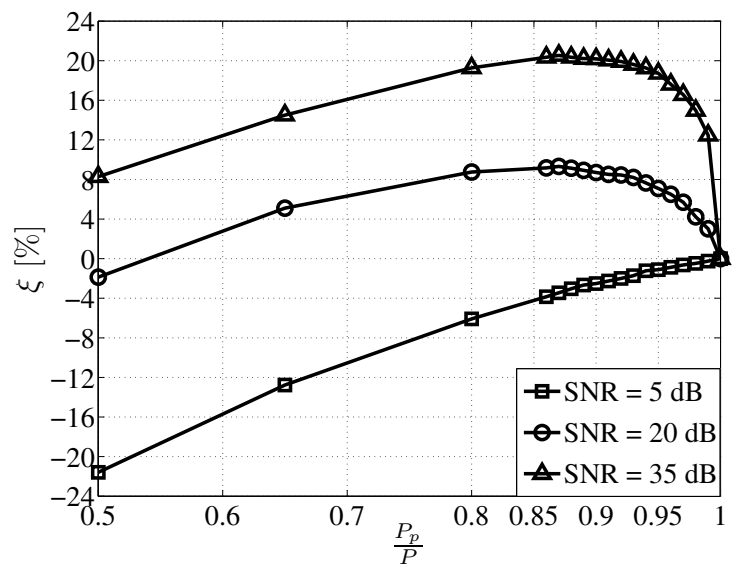

Fig. 3. Percent energy efficiency change w.r.t. the legacy OFDM femtocell for a Rayleigh fading channel, exponentially decreasing PDP.

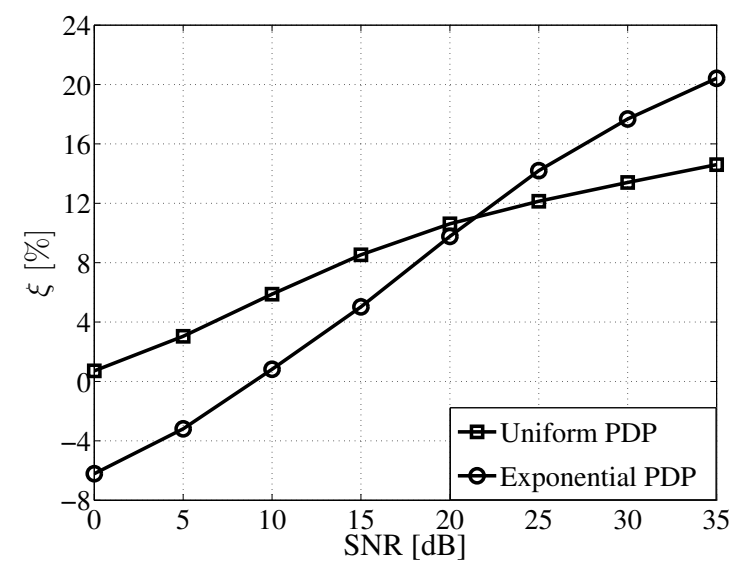

Fig. 4. Percentage of the maximum achievable spectral efficiency of CIA that can be achieved by the secondary transmission in the hybrid scheme.

the standalone OFDM transmission only for SNRs larger than $8 \mathrm{~dB}$. On the other hand, higher values for $\xi$ are achievable if compared to the uniform PDP case for SNRs larger than $21 \mathrm{~dB}$, exceeding the performance for the latter by about $6 \%$ at $35 \mathrm{~dB}$.

\section{CONCLUSIONS}

In this contribution, we have proposed a green approach to recycle unused resources of a legacy OFDM transmission with the goal to increase the spectral efficiency of femtocells. Since the total transmitted power remains constant, we effectively increase the energy efficiency as well. Additionally, we solve two critical issues of the underlying CIA technique: synchronization and channel knowledge acquisition. We show that, by adopting an MMSE linear equalizer, we are able to address one of the issues raised by the simultaneous transmission of primary and secondary messages, the sharing of a channel for both intended and interfering messages. Simulation results show that, by means of an appropriate power splitting strategy, energy efficiency gains of up to $15 \%$ are possible for uniform and exponential PDP channels, due to the CIA contribution. Furthermore, this finding corroborates our expectations that the power ratio should be shifted towards OFDM since it carries more information than CIA. Perspectives of this work include a detailed analysis of the optimal power splitting strategy and of the nature of the contributions from OFDM and CIA transmissions to the performance of the hybrid transmitter. Additionally, we will account for channel estimation errors at the transmitter and extend the network to a multi-femtocell layout, to move towards a more realistic operating scenario.

\section{ACKNOWLEDGMENTS}

This research has been supported by the ERC Starting Grant 305123 MORE (Advanced Mathematical Tools for Complex Network Engineering).

\section{REFERENCES}

[1] 3GPP. TR 36.814, Further advancements for E-UTRA physical layer aspects, v.9.0.0. Technical report, 3GPP, March 2010.

[2] Cisco. URL: http://www.cisco.com/en/US/solutions/collateral/ ns341/ns525/ns537/ns705/ns827/white_paper_c11-481360_ns827_ Networking_Solutions_White_Paper.html. Accessed: 31/12/2012.

[3] V. Chandrasekhar, J. Andrews, and A. Gatherer. Femtocell networks: a survey. IEEE Communications Magazine, 46(9):59-67, September 2008.

[4] D. Gesbert, S. Hanly, H. Huang, S. Shamai Shitz, O. Simeone, and Wei Yu. Multi-Cell MIMO Cooperative Networks: A New Look at Interference. IEEE Journal on Selected Areas in Communications, 28(9):1380-1408, December 2010.

[5] I.F. Akyildiz, W.Y. Lee, M.C. Vuran, and S. Mohanty. NeXt generation/dynamic spectrum access/cognitive radio wireless networks: a survey. Computer Networks, 50(13):2127-2159, 2006.

[6] The Climate Group and Global e-Sustainability Initiative (GeSI), "SMART 2020: Enabling the low carbon economy in the information age", URL: http://www.smart2020.org, 2008. Accessed: 31/12/2012.

[7] J. Hoydis, M. Kobayashi, and M. Debbah. Green Small-Cell Networks. IEEE Vehicular Technology Magazine, 6(1):37-43, March 2011.

[8] T. Chen, Y. Yang, H. Zhang, H. Kim, and K. Horneman. Network energy saving technologies for green wireless access networks. IEEE Wireless Communications, 18(5):30-38, October 2011.

[9] 3GPP. TR 36.902, Evolved Universal Terrestrial Radio Access Network (E-UTRAN); Self-configuring and self-optimizing network (SON) use cases and solutions, v.9.3.1. Technical report, 3GPP, May 2011.

[10] G. Miao, N. Himayat, and G. Y. Li. Energy-efficient link adaptation in frequency-selective channels. IEEE Transactions on Communications, 58(2):545-554, February 2010.

[11] M. Maso, L. S. Cardoso, M. Debbah, and L. Vangelista. Cognitive interference alignment for OFDM two-tiered networks. In IEEE 13th International Workshop on Signal Processing Advances in Wireless Communications (SPAWC), pages 244-248, June 2012.

[12] D. Tse and P. Viswanath. Fundamentals of Wireless Communication. Cambridge University Press, June 2005.

[13] L. S. Cardoso. Orthogonal Precoder for Dynamic Spectrum Access in Wireless Networks. PhD thesis, Supélec, November 2011.

[14] B. Hassibi and B.M. Hochwald. How much training is needed in multiple-antenna wireless links? IEEE Transactions on Information Theory, 49(4):951-963, April 2003.

[15] M. Debbah. Short introduction to OFDM. URL: flexible-radio.org/sites/ default/files/media/1/tutorial_ofdmtutorial.pdf. Accessed: 31/12/2012.

[16] M. Kobayashi, M. Debbah, and S. Shamai. Secured Communication over Frequency-Selective Fading Channels: a practical Vandermonde precoding. CoRR, abs/0906.3192, 2009. 